\title{
COMPORTAMENTO DE CLONES DE LARANJA 'VALÊNCIA' NA REGIÃO NORTE DO PARANÁ ${ }^{1}$
}

\author{
ZULEIDE HISSANO TAZIMA ${ }^{2}$, PEDRO ANTONIO MARTINS AULER ${ }^{3}$, CARMEN SILVIA VIEIRA JANEIRO NEVES $^{4}$, \\ INÊS FUMIKO UBUKATA YADA ${ }^{5}$, RUI PEREIRA LEITE JUNIOR ${ }^{6}$
}

RESUMO - Este trabalho teve como objetivo avaliar os clones de laranjas-doces [Citrus sinensis (L.) Osbeck] 'Valência', acesso I-93; 'Valência 718', acesso I-94, e 'Valência Late 1138', acesso I-105, enxertados sobre o limão 'Cravo' (Citrus limonia Osbeck), em relação à produção e às características físico-químicas dos frutos (acidez, sólidos solúveis totais, 'ratio', rendimento em suco, índice tecnológico e massa). As plantas estudadas fazem parte do Banco Ativo de Germoplasma de Citros (BAG-Citros) do Instituto Agronômico do Paraná - IAPAR, em Londrina. Foram utilizadas três plantas por clone, em espaçamento de 7,0 m x 6,0 m (238 plantas/hectare), conduzidas sem irrigação. As produções acumuladas das laranjas 'Valência' e 'Valência Late 1138', durante nove safras (1985 a 1994), foram significativamente superiores à da 'Valência 718'. Todos os clones apresentaram características aceitáveis de frutos, em relação à acidez, sólidos solúveis totais, 'ratio', rendimento em suco e massa do fruto, exceto para o índice tecnológico, que foi inferior na 'Valência Late 1138', não sendo observadas diferenças significativas durante o período avaliado (1986 a 1997).

Termos de indexação: Citrus sinensis, produção, qualidade do fruto.

\section{BEHAVIOUR OF VALENCIA ORANGE CLONES IN NORTHERN PARANÁ, BRAZIL}

ABSTRACT - The aim of this study was to evaluate clones of the sweet oranges [Citrus sinensis (L.) Osbeck] 'Valência' accession I-93, 'Valência 718' accession I-94 and 'Valência Late 1138' accession I-105, grafted on 'Rangpur' lime (Citrus limonia Osbeck), in relation to plant production, physical and chemical fruit characteristics (acidity, total soluble solids, TSS/TA ratio, juice content, technological index and fruit mass). The plants, three of each clone, were originated from the Citrus Active Germoplasm Bank (AGBCitrus) of the Instituto Agronômico do Paraná - IAPAR, located in Londrina, Paraná, Brazil. The plants were spaced at $7.0 \mathrm{~m} \times 6.0 \mathrm{~m}$ (238 plants/hectare) and maintained without irrigation. Cumulative yields of 'Valência' and 'Valência Late 1138' oranges, during nine crop seasons (1985 to 1994, except 1991) were significatively higher when compared to that of 'Valência 718' orange. All clones presented acceptable fruit quality in relation to acidity, total soluble solids, TSS/TA ratio, juice content and fruit mass, except for the technological index which was lower in 'Valência Late 1138'. There was no significant difference among the clones for these characteristics during 1986 to 1997 study period.

Index terms: Citrus sinensis, yield, fruit quality

\section{INTRODUÇÃO}

A citricultura tem grande importância para a fruticultura paranaense, ocupando uma área de 27.137 ha e produção de mais de 14 milhões de caixas (40,8 kg) em 2005 (Andretta, 2007). Entre as espécies cítricas, o cultivo da laranja é o que tem apresentado maior expansão, principalmente nas regiões norte e noroeste do Paraná. Vários fatores têm contribuído para esse aumento da área plantada, entre os quais as condições edafoclimáticas favoráveis para a citricultura, o estabelecimento de indústrias para o processamento de frutos visando à produção de suco concentrado congelado, preços remuneradores alcançados pelos produtores nas últimas safras, e também o potencial de crescimento dos mercados interno e externo.

No Paraná, são recomendadas para o plantio somente cultivares de citros que apresentem certo grau de resistência às doenças, principalmente ao cancro-cítrico causado pela bactéria Xanthomonas axonopodis, pv. citri. Seguindo esses critérios, estão recomendadas para plantio no Estado as laranjas-doces [Citrus sinensis (L.) Osbeck] 'Navelina', 'Shamouti', 'Salustiana', 'Cadenera', 'Jaffa', 'Pêra', 'IAPAR 73', 'Folha Murcha' e 'Valência' entre outras (Tazima \& Leite Júnior, 2002; Tazima \& Leite Júnior, 2000; Leite Júnior, 1992).

A laranja 'Valência' é uma cultivar de grande importância econômica devido à alta produtividade e qualidade dos frutos, sendo plantada nas principais regiões produtoras de citros do mundo. Essa cultivar apresenta maturação tardia dos frutos e

1(Trabalho 276-07). Recebido em: 21-11-2007. Aceito para publicação em: 10-04-2008.

${ }^{2} E_{n g}{ }^{\text {Agra }}{ }^{\text {, }}$ Doutoranda, Pesquisadora, Instituto Agronômico do Paraná - IAPAR, Área de Ecofisiologia, C.P. 481, CEP 86001-970 - Londrina-PR. Email: zuleide@iapar.br

${ }^{3}$ Eng $^{\text {o. }}$ Agr $^{\circ}$, Doutorando, Pesquisador, Instituto Agronômico do Paraná - IAPAR, Área de Fitotecnia, C.P. 481, CEP 86001-970 - Londrina-PR. E-mail: aulerpe@iapar.br

${ }^{4}$ Eng $^{\text {a }}$ Agra $^{a}$, Dr ${ }^{\text {a }}$, Professora, Universidade Estadual de Londrina-UEL, Departamento de Agronomia, C.P. 6001 - CEP $86051-990$ - Londrina-PR. Bolsista em Produtividade do Conselho Nacional de Desenvolvimento Científico e Tecnológico-CNPq). E-mail: csvjneve@uel.br

${ }_{5}^{5}$ Lic. Matemática, M. Sc., Pesquisadora, Instituto Agronômico do Paraná - IAPAR, Área de Biometria, C.P. 481, CEP 86001-970 - Londrina-PR. Email: inesyada@iapar.br

${ }^{6} \mathrm{Eng}^{\circ} \mathrm{Agr}^{\circ}$, Dr, Pesquisador, Instituto Agronômico do Paraná - IAPAR, Área de Proteção de Plantas, C.P. 481, CEP 86001-970 - Londrina-PR. E-mail: ruileite@iapar.br

Rev. Bras. Frutic., Jaboticabal - SP, v. 30, n. 4, p.970-974, Dezembro 2008 
pode ser destinada tanto para o mercado interno como para o externo, atendendo ao consumo de fruta fresca e ao processamento industrial. É originária provavelmente de Portugal e foi introduzida no Brasil a partir de material propagativo importado da Flórida (Leite Júnior, 1992). Conforme estudos realizados no Instituto Agronômico do Paraná - IAPAR, essa cultivar é considerada como moderadamente resistente ao cancrocítrico (Leite Júnior, 1992).

Uma das principais metas do melhoramento de citros é buscar cultivares produtivas, e para isso é necessário o estudo do comportamento desse material em diferentes localidades (Domingues et al., 1999). O comportamento de cada cultivar ou clone de citros está associado às condições edafoclimáticas locais, que devem proporcionar às plantas um ambiente para que exerçam sua máxima capacidade genética, resultando nos melhores rendimentos econômicos (Rodriguez, 1987).

O IAPAR, instituição responsável pelo material genético da citricultura paranaense, possui um Banco Ativo de Germoplasma de Citros (BAG-Citros) onde os diferentes genótipos se encontram em avaliação para a determinação da adaptação às condições edafoclimáticas do Paraná. Embora as características botânicas e agronômicas da laranja 'Valência' já sejam conhecidas (Hodgson, 1967), faltam ainda informações sobre o comportamento da cultivar nas condições do norte do Estado. Portanto, o objetivo deste trabalho foi estudar o comportamento de três clones da laranja 'Valência' enxertados sobre limão 'Cravo' (Citrus limonia Osbeck), em relação à produção e características físicas e químicas dos frutos.

\section{MATERIAL E MÉTODOS}

O estudo foi desenvolvido na Estação Experimental do IAPAR, no município de Londrina-PR, em Latossolo Vermelho distroférrico a uma altitude de $585 \mathrm{~m}$, latitude $23^{\circ} 22^{\prime}$ S e longitude $51^{\circ} 10^{\prime} \mathrm{W}$. O clima da região é do tipo Cfa - clima subtropical, com verões quentes, geadas pouco freqüentes e tendência de concentração das chuvas nos meses de verão, contudo sem estação seca definida. As temperaturas médias, da máxima e mínima, são $27,3^{\circ} \mathrm{Ce} 16^{\circ} \mathrm{C}$, respectivamente; a precipitação média anual é de $1.588 \mathrm{~mm}$, e a umidade relativa média é de 70,6\% (Figura 1) (INSTITUTO..., 2007).

Foram avaliados três acessos de laranja 'Valência' enxertados sobre limão 'Cravo' pertencentes ao BAG-Citros do IAPAR: 'Valência', acesso I-93, proveniente da coleção de citros da Faculdade de Ciências Agrárias da Universidade Estadual Paulista-UNESP/Botucatu - SP; 'Valência 718' e 'Valência Late 1138', acessos I-94 e I-105, respectivamente, provenientes da coleção de citros do Centro Avançado de Pesquisa Tecnológica do Agronegócio de Citros Sylvio Moreira (CAPTACSM), vinculado ao Instituto Agronômico de Campinas - IAC/ Cordeirópolis-SP. As plantas, em número de três de cada introdução, foram plantadas em fevereiro de 1982, em espaçamento de $7,0 \mathrm{~m} \times 6,0 \mathrm{~m}$, correspondendo a 238 plantas por hectare e cultivadas sem irrigação. Os tratos culturais foram realizados segundo as recomendações técnicas para o Estado do Paraná (INSTITUTO..., 1992).
Durante o período de 1985 a 1994, exceto 1991, foi avaliada a produção de frutos por planta, e de 1986 a 1997, as características físico-químicas dos frutos. Para isso, por ocasião da colheita, foram realizadas a contagem e a pesagem dos frutos para a determinação da produção média por planta e da massa média do fruto. A produção acumulada foi obtida somando-se a produção média anual dos nove anos avaliados. Para a análise química, foram coletadas amostras contendo 10 frutos da parte externa da planta, à altura de 1,0 m a 2,0 m, nos quatro quadrantes. A extração do suco foi realizada com extratora Croydon ${ }^{\circledR}$, modelo ES4EAB60000. A acidez titulável total (ATT) foi determinada pela titulação de $25 \mathrm{~mL}$ de suco, com solução de hidróxido de sódio a 0,1 N (AOAC, 1990), obtendo-se o resultado em porcentagem de ácido cítrico. O teor de sólidos solúveis totais (SST) foi determinado pela leitura direta em refratômetro Atago ${ }^{\circledR}$. O 'ratio', que é a relação aritmética entre sólidos solúveis totais e acidez (SST/ATT), foi calculado obtendo-se um valor aproximado do ponto de maturação dos frutos, pois os dados obtidos para análise foram coletados de agosto a outubro.

$\mathrm{O}$ rendimento em suco, expresso em porcentagem, foi calculado através da relação: (MS/MF) x 100, onde MS = massa do suco (g) e MF = massa da fruta (g). O índice tecnológico (IT) ou quantidade de sólidos solúveis totais no suco, em uma caixa de 40,8 $\mathrm{kg}$ ( $\mathrm{kg}$ de SST.caixa $\left.{ }^{-1}\right)$, foi calculado conforme a fórmula abaixo (Di Giorgi et al., 1990):

IT $=$ [rendimento em suco $\mathrm{x}$ sólidos solúveis totais $\mathrm{x} 40,8$ ] x $10.000^{-1}$, onde:

IT = índice tecnológico; rendimento em suco = relação MS/MF; sólidos solúveis totais = teor de sólidos solúveis; 40,8 $\mathrm{kg}$ = peso-padrão da caixa de colheita de laranja.

Os dados foram analisados utilizando-se do programa SAS (SAS INSTITUTE INC., 2001) para a comparação das médias, teste $\mathrm{t}$, a $5 \%$ de probabilidade.

\section{RESULTADOS E DISCUSSÃO}

A produção de frutos do clone 'Valência' foi superior à 'Valência 718', em 1985, 1988, 1990 e 1994, e à 'Valência Late 1138', em 1985 (Tabela 1). Este último clone foi superior à 'Valência 718', em 1988, 1989 e 1994, sendo também superior ao clone 'Valência', em 1989. Os três clones comportaram-se de modo semelhante, em 1986, 1987, 1992 e 1993, não diferindo estatisticamente. Considerando a produção acumulada nos nove anos de colheita, as laranjas 'Valência' e 'Valência Late 1138' apresentaram os maiores valores, diferindo estatisticamente da 'Valência 718'. Auler et al. (2008) registraram produção acumulada de $867,69 \mathrm{~kg}$ de frutos por planta nas dez primeiras safras (1996 a 2005) para o clone 'Valência 718', na região noroeste do Estado do Paraná.

A produção média anual dos três clones de 'Valência', durante o período de três a cinco anos após o plantio (1985 a 1987), variou de $48,27 \mathrm{~kg}$ a $68,27 \mathrm{~kg}$ por planta (Tabela 2). Entretanto, essa produtividade ficou abaixo da obtida por Roberto et al. (1999), que registrou produção média de $96,77 \mathrm{~kg}$, para período de produção equivalente nas condições de Santa Rita do Passa Quatro-SP. A produção inicial observada em 1984 foi 
pequena e sem expressão econômica. Conforme Di Giorgi et al. (1990), uma planta de laranja torna-se produtiva a partir de 4 a 5 anos de idade. A produção média por planta dos três acessos de laranja 'Valência' obtida no período compreendido entre 1985 a 1990 variou de $95,60 \mathrm{~kg}$ a 131,48 $\mathrm{kg}$ (Tabela 2), superando os resultados obtidos por Souto et al. (2001), em Nova Porteirinha (MG), com os clones 'Valência CNPMF 27'e 'Valência IAC 36', que foram de 90 e $58 \mathrm{~kg}$ por planta, respectivamente.

No período de 1992 a 1994 (10 a 12 anos a partir do plantio), a produção média dos três clones de 'Valência' variou de 157,14 $\mathrm{kg}$ a 264,38 kg (Tabela 2), estando as produções das laranjas 'Valência'e 'Valência Late 1138' dentro da média estimada de 200 $\mathrm{kg}$ por planta para essa cultivar (Figueiredo, 1991). A laranja 'Valência 718' apresentou produção relativamente baixa quando comparada com a média esperada de $200 \mathrm{~kg}$ por planta. No entanto, Pompeu Jr. (1981) relatou que, no total de dez safras (1970 a 1979), foi obtida produção média de $160,8 \mathrm{~kg}$ para a laranja 'Valência', nas condições da Estação Experimental de Pindorama do IAC - SP.

Na produção obtida na fase adulta, de 1992 a 1994 (Tabela 1), foi observada tendência à alternância de produção para os três clones de laranja 'Valência'. Jones \& Cree (1965) demonstraram, experimentalmente, que a coexistência de frutos em plena maturidade e flores do ciclo posterior acarreta variações na produção, provocando a bianualidade. Isso é verificado normalmente nas cultivares tardias (Di Giorgi et al., 1993).

As variáveis avaliadas acidez, sólidos solúveis, 'ratio', rendimento em suco e massa do fruto, não apresentaram diferenças significativas entre os três clones estudados, exceto para o índice tecnológico da 'Valência Late 1138', cujo valor de $1,95 \pm 0,24 \mathrm{~kg}$ de SST.caixa ${ }^{-1}$ foi inferior aos demais $(2,20 \pm 0,32$ para 'Valência 718' e 2,16 \pm 0,35 para 'Valência') (Tabela 3). Segundo Di Giorgi et al. (1990), este índice varia de 2,49 a 2,86 kg de SST.caixa ${ }^{-1}$.

O valor médio de sólidos solúveis totais (SST) variou de 9,81 $\pm 1,02$ a $11,03 \pm 1,43^{\circ}$ Brix, e, segundo Figueiredo (1991), a média deste índice para esta cultivar é de 11,8. Os menores valores de SST obtidos, quando comparados aos padrões da cultivar, são devido à colheita antecipada dos frutos (agosto a outubro).

Os valores de acidez variaram de $1,19 \pm 0,24$ a $1,28 \pm$ $0,31 \%$, enquanto Figueiredo (1991) descreveu 1,05 para laranja 'Valência'. Esses valores de acidez também estão relacionados com o período de colheita, indicando que os frutos ainda não haviam completado a maturação. Conforme Chitarra \& Chitarra (2005), a acidez dos frutos tende a decrescer com a utilização dos ácidos orgânicos na atividade respiratória, que é intensa à medida que segue o crescimento e a maturação dos frutos. Portanto, colheitas mais tardias permitiriam a maturação completa dos frutos, favorecendo um incremento nos valores de 'ratio' obtidos que variaram de $8,43 \pm 1,43$ a $8,51 \pm 1,68$. Segundo Di Giorgi et al. (1993), enquanto o fruto permanece na árvore, sua qualidade interna aumenta gradativamente, atingindo um valor máximo para a fabricação do suco de laranja concentrado congelado, situado na faixa de ratio 13 a 16. Em avaliações realizadas em meados de outubro no noroeste do Paraná, Auler et al. (2008) obtiveram 'ratio' médio de 13,4 para a laranja 'Valência 718'.
Orendimento em suco variou de 48,90 $\pm 3,18$ para 'Valência Late 1138 ' a 50,87 \pm 4,51 para 'Valência'. Figueiredo (1991) considera que o rendimento da cultivar Valência é de $50 \%$. A massa média dos frutos nos três tratamentos foi superior à média da cultivar, que é de $150 \mathrm{~g}$ (Figueiredo, 1991), aproximando-se dos 184,5 g relatados por Pompeu Júnior (1981) e dos 182,0 g relatados por Auler et al. (2008), e inferior aos $230 \mathrm{~g}$ obtidos por Souto (2001).

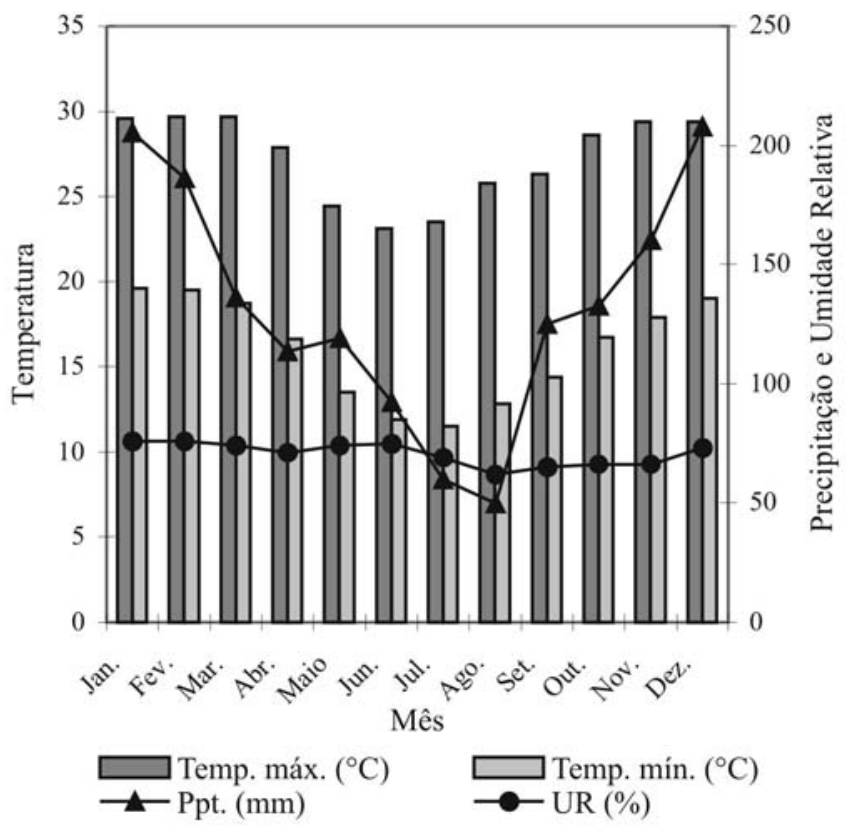

FIGURA 1 - Médias das temperaturas máxima e mínima, precipitação e umidade relativa da Estação Experimental do Instituto Agronômico do Paraná - IAPAR, Londrina - PR, para o período de 1976 a 2006.

TABELA 1 - Produção média anual e total acumulada $\left(\mathrm{kg} \cdot\right.$ planta $\left.^{-1}\right)$ de nove safras de três clones de laranja 'Valência' do BAG-Citros do IAPAR, Londrina-PR, de 1985 a 1994*.

\begin{tabular}{crrrrrr}
\hline $\begin{array}{c}\text { Ano da } \\
\text { colheita }\end{array}$ & 'Valência' & $\begin{array}{c}\text { Clone } \\
\text { 'Valência } \\
718\end{array}$ & $\begin{array}{r}\text { 'Valência Late } \\
1138\end{array}$ \\
\hline 1985 & $41,85^{z}$ & a & 3,10 & $\mathrm{~b}$ & 7,55 & $\mathrm{~b}$ \\
1986 & 15,20 & $\mathrm{a}$ & 6,74 & $\mathrm{a}$ & 4,84 & $\mathrm{a}$ \\
1987 & 147,77 & $\mathrm{a}$ & 124,99 & $\mathrm{a}$ & 141,30 & $\mathrm{a}$ \\
1988 & 237,08 & $\mathrm{a}$ & 149,35 & $\mathrm{~b}$ & 256,59 & $\mathrm{a}$ \\
1989 & 189,04 & $\mathrm{~b}$ & 199,94 & $\mathrm{~b}$ & 267,95 & $\mathrm{a}$ \\
1990 & 126,83 & $\mathrm{a}$ & 79,50 & $\mathrm{~b}$ & 110,67 & $\mathrm{ab}$ \\
1992 & 215,03 & $\mathrm{a}$ & 168,43 & $\mathrm{a}$ & 268,17 & $\mathrm{a}$ \\
1993 & 311,37 & $\mathrm{a}$ & 241,10 & $\mathrm{a}$ & 313,77 & $\mathrm{a}$ \\
1994 & 190,13 & $\mathrm{a}$ & 61,90 & $\mathrm{~b}$ & 211,20 & $\mathrm{a}$ \\
Total & & & & & & \\
acumulado & $1.474,30$ & $\mathrm{a}$ & $1.045,05$ & $\mathrm{~b}$ & $1.582,04$ & $\mathrm{a}$ \\
\hline
\end{tabular}

${ }^{2}$ Médias seguidas da mesma letra na linha não diferem entre si, ao nível de $5 \%$ de probabilidade, pelo teste $\mathrm{t}$

*1991 - dados não coletados. 
TABELA 2 - Produção média (kg.planta-1) de três clones de laranja Valência' do BAG - Citros do IAPAR, Londrina-PR, de 1985 a 1994.

\begin{tabular}{crrr}
\hline $\begin{array}{c}\text { Ano da } \\
\text { Colheita* }\end{array}$ & 'Valência' & $\begin{array}{c}\text { Clone } \\
\text { 'Valência } \\
718\end{array}$ & $\begin{array}{c}\text { 'Valência Late } \\
1138\end{array}$ \\
\hline 1985 a 1987 & $68,27^{2}$ a & 48,27 a & 51,23 a \\
1988 a 1990 & 184,32 a & 142,93 a & 211,74 a \\
1992 a 1994 & 238,84 a & 157,14 a & 264,38 a \\
1985 a 1990 & 126,30 a & $95,60 \mathrm{a}$ & $131,48 \mathrm{a}$ \\
\hline
\end{tabular}

${ }^{z}$ Médias seguidas da mesma letra na linha não diferem entre si ao nível de $5 \%$ de probabilidade, pelo teste $\mathrm{t}$.

*1991 - dados não coletados

TABELA 3 - Dados médios de acidez total (AT), sólidos solúveis totais (SST), 'Ratio’ (SST/AT), rendimento em suco (Suco), índice tecnológico (IT) e massa do fruto (MF) de três clones de laranja 'Valência' do BAG-Citros do IAPAR, Londrina, 1986 a 1997.

\begin{tabular}{ccccccc}
\hline \multicolumn{1}{c}{ Clone } & $\begin{array}{c}\text { AT } \\
(\%)\end{array}$ & $\begin{array}{c}\text { SST } \\
\left({ }^{\circ} \text { Brix }\right)\end{array}$ & $\begin{array}{c}\text { 'Ratio' } \\
\text { SST/AT }\end{array}$ & $\begin{array}{c}\text { Suco } \\
(\%)\end{array}$ & $\begin{array}{c}\text { IT } \\
\text { SST.caixa }\end{array}$ & $\begin{array}{c}\text { MF }(\mathrm{kg}) \\
(\mathrm{g})\end{array}$ \\
\hline 'Valência' & $1,28^{\mathrm{z}} \mathrm{a}$ & $10,48 \mathrm{a}$ & $8,51 \mathrm{a}$ & $50,87 \mathrm{a}$ & $2,16 \mathrm{a}$ & $169,88 \mathrm{a}$ \\
'Valência 718' & $1,33 \mathrm{a}$ & $11,03 \mathrm{a}$ & $8,43 \mathrm{a}$ & $49,06 \mathrm{a}$ & $2,20 \mathrm{a}$ & $174,67 \mathrm{a}$ \\
'Valência Late $1138 '$ & $1,19 \mathrm{a}$ & $9,81 \mathrm{a}$ & $8,48 \mathrm{a}$ & $48,90 \mathrm{a}$ & $1,95 \mathrm{~b}$ & $183,16 \mathrm{a}$ \\
\hline
\end{tabular}

${ }^{2}$ Médias seguidas da mesma letra na coluna não diferem entre si ao nível de 5\% de probabilidade, pelo teste t.

\section{CONCLUSÕES}

1-Nas condições da região norte do Paraná, os clones de laranja 'Valência', acesso I-93, e 'Valência Late 1138', acesso I105, são os mais produtivos em relação ao clone 'Valência 718', acesso I-94

2-A laranja 'Valência Late 1138', acesso I-105, apresenta o menor índice tecnológico em relação aos demais clones para o período avaliado (agosto a outubro).

\section{AGRADECIMENTOS}

Agradecemos aos colaboradores Sydnei Dias dos Santos, Naodi Komoli e José Antonio de Oliveira, pelo apoio prestado na condução dos trabalhos e avaliações.

\section{REFERÊNCIAS}

ANDRETTA, G. M. A. C. Valor bruto da produção agropecuária paranaense de 2005. Curitiba, SEAB/DERAL/DEB, 2007. 84 p.

AOAC - ASSOCIATION OF OFFICIAL ANALYTICAL CHEMISTS. Official methods of analysis. $15^{\text {th }}$ ed. Arlington: AOAC, 1990. $1.298 \mathrm{p}$.

AULER, P. A. M.; FIORI-TUTIDA, A. C. G.; TAZIMA, Z. H. Comportamento da laranjeira 'Valência' sobre seis porta-enxertos no noroeste do Paraná. Revista Brasileira de Fruticultura, Jaboticabal, v.30, n.1, p.229-234, mar.2008.

CHITARRA, M. I. F.; CHITARRA, A. B. Pós-colheita de frutos e hortaliças: fisiologia e manuseio. 2. ed. Lavras: UFLA, 2005.
DI GIORGI, F. et al. Contribuição ao estudo do comportamento de algumas variedades de citros e suas implicações agroindustriais. Laranja, Cordeirópolis, v. 11, n. 2, p. 567-612, 1990.

DI GIORGI, F. et al. Qualidade da laranja para industrialização. Laranja, Cordeirópolis, v. 11, n. 1, p. 97-118, 1993.

DOMINGUES, E. T. et al. Seleção de clones de laranja 'Pêra' e variedades assemelhadas quanto à qualidade do fruto e ao período de maturação. Laranja, Cordeirópolis, v. 20, n. 2, p. 433$455,1999$.

FIGUEIREDO, J. O. Variedades comerciais. In: RODRIGUEZ, O.: VIÉGAS, F.; POMPEU JR., J.; AMARO, A. A. Citricultura brasileira. 2. ed. Campinas: Fundação Cargill, 1991. v. 1, p. 22857.

HODGSON, R. W. Horticultural Varieties of Citrus. In: REUTHER, W. et. al. (Eds). The Citrus industry. California: University of California, 1967.p. 431-588.

IAPAR - INSTITUTO AGRONÔMICO DO PARANÁ. A citricultura no Paraná. Londrina, 1992. 288 p. (Circular 72).

IAPAR - INSTITUTO AGRONÔMICO DO PARANÁ, Londrina, PR. Monitoramento agroclimático do Paraná. Disponível em: <http://200.201.27.14/Site/Sma/Estacoes_IAPAR/ Estacoes_Parana.htm>. Acesso em: 27 abr. 2007.

JONES, W. W.; CREE, C. B. Environmental factors related to fruiting of Washington Navel oranges over a 38-year period. Proceedings of the American Society for Horticultural Science, Beltsville, v. 86, p. 267-271, 1965. 
LEITE JR, R. P. L. Cultivares de copa e porta-enxertos. In: IAPAR. A citricultura no Paraná. Londrina: IAPAR, 1992. p. 91-116. (Circular, 72).

POMPEU JR, J. Comportamento da laranjeira-Valência Citrus sinensis (L.) Osbeck, em 18 porta-enxertos. In: CONGRESSO BRASILEIRO DE FRUTICULTURA, 6., 1981, Recife. Anais... Recife: Sociedade Brasileira de Fruticultura, 1981. p. 530-541.

ROBERTO, S. R.; LIMA, J. E. O.; CARLOS, E. F. Produtividade inicial da laranjeira 'Valência' (Citrus sinensis L. Osbeck): sobre oito porta-enxertos no Estado de São Paulo. Revista Brasileira de Fruticultura, Jaboticabal, v. 21, n. 2, p. 119-122, ago. 1999.

RODRIGUEZ, O. Ecofisiologia dos citros. In: CASTRO, P.R.C. (Ed.). Ecofisiologia da produção agrícola. Piracicaba: Associação Brasileira para Pesquisa da Potassa e do Fosfato, 1987. p. 149164.
SAS INSTITUTE. SAS/STAT user's Guide. Version 8.2. Cary, NC, $2001.943 \mathrm{p}$.

SOUTO, R.F. et al. Comportamento de laranjeiras-doces no norte do Estado de Minas Gerais. Laranja, Cordeirópolis, v. 22, n. 2, p. 457-467, 2001.

TAZIMA, Z. H.; LEITE JÚNIOR, R. P. Novos cultivares de citros para o Paraná. In: CONGRESSO BRASILEIRO DE FRUTICULTURA, 17., 2002, Belém. Anais... Belém: Sociedade Brasileira de Fruticultura, 2002. CD-ROM.

TAZIMA, Z. H.; LEITE JÚNIOR, R. P. IAPAR-73: NOVA CULTIVAR PRECOCE DE LARANJA (Citrus sinensis (L.) Osb.) para o Estado do Paraná. In: CONGRESSO BRASILEIRO DE FRUTICULTURA, 16., 2000, Fortaleza. Anais... Fortaleza: Sociedade Brasileira de Fruticultura, 2000. CD-ROM. 